\title{
Decreased expression of microRNA-126 is associated with poor prognosis in patients with cervical cancer
}

\author{
Yao Yang ${ }^{1}$, Kun-ling Song ${ }^{2}$, Hong Chang $^{3}$ and Long Chen ${ }^{1 *}$
}

\begin{abstract}
Background: MicroRNA-126(miR-126) has been shown to be frequently down-regulated in a variety of malignancies and act as a potential tumor suppressor. However, its correlations with the clinicopathological characters of cervical cancer remain unclear.

Methods: TaqMan quantitative RT-PCR was used to determine the expression level of miR-126 in tissue samples. The associations of miR-126 expression with clinicopathologic variables were analyzed. Kaplan-Meier survival analysis was performed to analyze the association of miR-126 expression with overall survival (OS) of patients. Univariate and multivariate Cox regression analyses were performed.

Results: miR-126 expression level in human cervical cancer tissues was significantly lower than that in adjacent nontumorous tissues (mean \pm SD: $0.59 \pm 0.44$ vs. $1.00 \pm 0.51, P<0.0001$ ). Decreased miR-126 expression in cervical cancer was found to be significantly associated with lymphatic invasion $(P=0.002)$, distant metastasis $(P<0.001)$, FIGO stage $(P=0.009)$, and histological grade $(P=0.005)$. Kaplan-Meier analysis showed that patients with lower levels of miR-126 had significantly poorer survival than those with higher expression of this miRNA in patients, with a 5 -year OS of $45.7 \%$ and $70.9 \%$, respectively $(P=0.002)$. Multivariate analysis revealed that miR-126 expression ( $\mathrm{HR}=3.97,95 \% \mathrm{Cl}: 2.01-20.22 ; P=0.003)$ was independently associated with the OS.
\end{abstract}

Conclusion: Our data suggests the potential of miR-126 as a prognostic biomarker for cervical cancer.

Virtual Slides: The virtual slide(s) for this article can be found here: http://www.diagnosticpathology.diagnomx.eu/ vs/13000_2014_220

Keywords: miRNA, miR-126, Cervical cancer, Quantitative RT-PCR, Prognosis

\section{Background}

Cervical cancer is the second leading cause of death among women worldwide, with an estimated 530,000 deaths per year [1]. Although radiotherapy, chemotherapy and surgery have been recently used as standard treatment modalities for patients with cervical cancer, with consequent disease remission, clinical outcomes vary significantly between patients and can be difficult to predict. Therefore, it is important to understand the complete knowledge of the molecular biology, genetics, causes and cellular origin of cervical cancers which are of value in the

\footnotetext{
* Correspondence: qingdao_chenlong@126.com

'Department of Gynecology, Qingdao Municipal Hospital, No.1 Jiaozhou Road, Qingdao 266011, People's Republic of China

Full list of author information is available at the end of the article
}

development of improved therapeutic strategies and in the identification of prognostic markers [2].

MicroRNAs (miRNAs) are small, conserved, noncoding short RNAs of 18-25 nucleotides in length that bind to target mRNAs mainly at their 3'-untranslated region (UTR) [3]. Many miRNAs have been implicated as key regulators of cellular growth and differentiation and have been found to deregulate proliferation in human cancers [4-6]. In human cancers, the expression of miRNAs is generally down-regulated or up-regulated in malignant tissues compared with the corresponding nonmalignant tissues, suggesting the deregulation of miRNA expression and the contribution of miRNAs to the multistep processes of carcinogenesis, either as oncogenes or as tumor-suppressor genes [7-9]. 
MiR-126 is frequently down-regulated in a variety of malignancies and acts as a potential tumor suppressor [10-12]. Moreover, low expression of miR-126 has been found to be correlated with poor prognosis in patients with breast cancer, adult $\mathrm{T}$ cell leukemia, colorectal cancer(CRC) and malignant mesothelioma [13-15]. Previously, miR-126 expression level in cervical cancer tissues was found to be significantly decreased compared with that in normal cervical tissues $(P<0.01)$. However, the clinical significance and prognostic value of miR-126 in cervical cancer have not been investigated.

\section{Methods}

\section{Patients and tissue samples}

Fresh cervical cancer and matched adjacent normal tissue specimens were collected from 133 patients who underwent surgery between March 2008 and July 2013 in the Department of Gynecology, Qingdao Municipal Hospital. The corresponding adjacent normal tissues were obtained $3 \mathrm{~cm}$ beyond the boundary of cervical cancer tissues. The fresh tissue specimens were immediately frozen in liquid nitrogen until use. The selection criteria for patients with cervical cancer were as follows: (1) pathologically confirmed patients with cervical cancer; (2) the patients had no history of other cancers. No patients had preoperative chemotherapy, radiotherapy, or other treatment history or other inflammatory diseases. Patient's conditions were staged according to the criteria of the International Federation of Gynecology and Obstetrics (FIGO). The clinicopathologic features of all the patients were summarized in Table 1 . Overall survival(OS) time was calculated from the date of the initial surgical operation to death. Follow-up information of all patients was updated every 3 months for the first 2 years, every 4 months for the third year, every 6 months for the fourth and fifth years, and then every year thereafter by telephone visit and questionnaire letters. Death of the participants was ascertained by reporting from the family and verified by review of public records.

This study was approved by the Research Ethics Committee of the Qingdao Municipal Hospital. Written informed consent was obtained from all of the patients.

All specimens were handled and made anonymous according to the ethical and legal standards.

\section{RNA extraction and Real-Time RT-PCR}

Total RNA was extracted with Trizol reagent (Invitrogen, Carlsbad, CA, USA) according to the manufacturer's instructions. The concentration and purity of all RNA samples were detected by NanoDrop ND-2000 spectrophotometer (NanoDrop Technologies, Houston, TX, USA). NCode ${ }^{\text {tw }}$ SYBR $^{\circ}$ Green miRNA qRT-PCR Kit (Invitrogen, Carlsbad, CA, USA) was used to synthesize specific cDNA of miR-126 and U6B(as an internal
Table 1 The relationship between miR-126 expression and clinicopathological characteristics in 133 patients with cervical cancer

\begin{tabular}{|c|c|c|c|c|}
\hline \multirow[b]{2}{*}{ Parameters } & \multirow{2}{*}{$\begin{array}{l}\text { Number } \\
\text { of cases }\end{array}$} & \multicolumn{3}{|c|}{ miR-126 expression } \\
\hline & & Low & High & $P$ value \\
\hline \multicolumn{5}{|l|}{ Age (y) } \\
\hline$<65$ & 56 & 29 & 27 & 0.46 \\
\hline$\geq 65$ & 77 & 33 & 44 & \\
\hline \multicolumn{5}{|l|}{ HPV } \\
\hline$(+)$ & 97 & 47 & 50 & 0.21 \\
\hline$(-)$ & 36 & 15 & 21 & \\
\hline \multicolumn{5}{|l|}{ Tumor histology } \\
\hline Squamous & 101 & 54 & 47 & 0.19 \\
\hline Adenocarcinoma & 22 & 5 & 17 & \\
\hline Clear cell & 10 & 3 & 7 & \\
\hline \multicolumn{5}{|l|}{ Tumor Size (cm) } \\
\hline$<4$ & 54 & 19 & 35 & 0.07 \\
\hline$\geq 4$ & 79 & 43 & 36 & \\
\hline \multicolumn{5}{|l|}{ Lymphatic invasion } \\
\hline Yes & 63 & 45 & 18 & 0.002 \\
\hline No & 70 & 17 & 53 & \\
\hline \multicolumn{5}{|l|}{ Distant metastasis } \\
\hline Yes & 28 & 23 & 5 & $<0.001$ \\
\hline No & 105 & 39 & 66 & \\
\hline \multicolumn{5}{|l|}{ FIGO stage } \\
\hline$|/| \mid$ & 62 & 20 & 42 & 0.009 \\
\hline III/IV & 71 & 42 & 29 & \\
\hline \multicolumn{5}{|l|}{ Histological grade } \\
\hline Well/moderate & 82 & 25 & 57 & 0.005 \\
\hline Poor & 51 & 37 & 14 & \\
\hline
\end{tabular}

control), and perform qRT-PCR, which was analyzed with the DNA Engine Opticon 2 Real-Time Cycler (MJ Research Inc., Waltham, MA, USA) according to the manufacturer's instructions. Each sample was examined in triplicate and analyzed by the comparative threshold cycle $(\mathrm{Ct})$ method. The expression levels of miR-126 were normalized to U6B.

\section{Statistical analysis}

Statistical analysis was conducted using the SPSS 18.0 for Windows (SPSS Inc., Chicago, IL, USA). The chisquare test was used to assess miR-126 expression with respect to clinicopathological parameters. The survival curves of the patients were determined using the Kaplan-Meier method and Cox regression, and the logrank test was used for statistical evaluations. Univariate Cox regression was performed on each clinical covariate to examine its influence on patient survival. Final 
multivariate models were based on step-wise addition. A Wald statistic of $P<0.05$ was used as the criterion for inclusion in final multivariate models. Data were expressed as the mean and standard deviation and analyzed using one-way analysis of variance. $P<0.05$ was considered to indicate a significant difference.

\section{Results}

The expression level of miR-126 in cervical cancer qRT-PCR was used to assess the expression of miR-126 in cervical cancer tissues and adjacent non-tumorous tissues. The results showed that miR-126 expression level in human cervical cancer tissues was significantly lower than that in adjacent nontumorous tissues (mean $\pm \mathrm{SD}: \quad 0.59 \pm 0.44$ vs. $\quad 1.00 \pm 0.51, \quad P<0.0001$; shown in Figure 1).

\section{Correlation of miR-126 expression with clinicopathological characteristics}

The Median expression level of miR-126 was used as a cut-off point to divide all 133 patients into two groups: cervical cancer patients who expressed miR-126 at levels less than the cut-off value were assigned to the low expression group ( $n=62$ ), and those with expression above the cut-off value were assigned to the high expression group ( $\mathrm{n}=71)$. The relationships between miR-126 expression levels and different clinicopathological factors were shown in Table 1. Decreased miR-126 expression in cervical cancer was found to be significantly associated with lymphatic invasion $(P=0.002)$, distant metastasis $(P<0.001)$, FIGO stage $(P=0.009)$, and histological grade $(P=0.005)$. However, no significant correlation was observed between miR-126 expression and other clinicopathologic variables, such as age, HPV infection, tumor histology, and tumor size (all $P>0.05$ ).

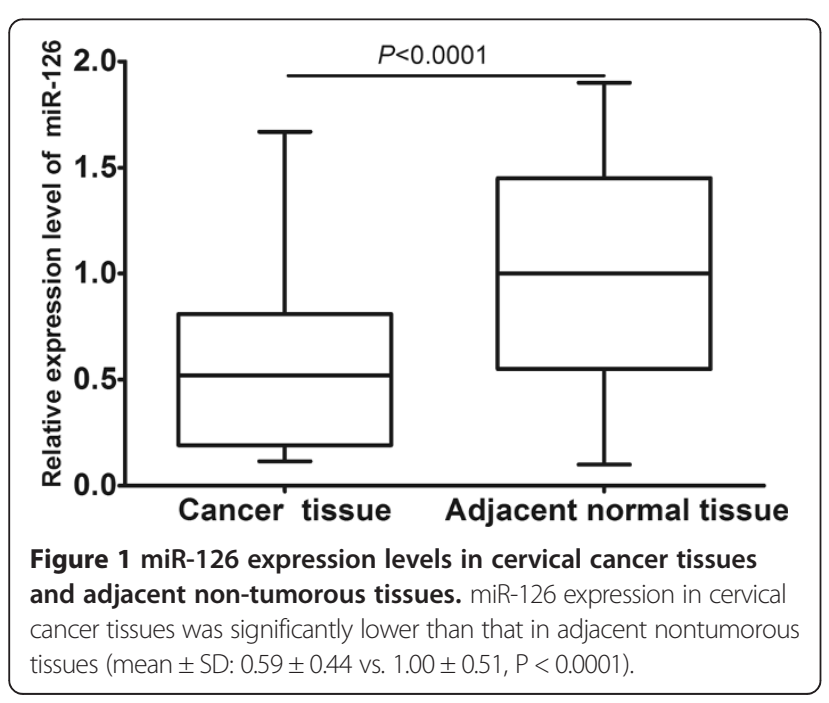

\section{Relationship between miR-126 expression and cervical cancer patients' survival}

To evaluate whether miR-126 expression can predict cervical cancer prognosis, we next performed survival analysis. Kaplan-Meier analysis showed that patients with lower levels of miR-126 had significantly poorer survival than those with higher expression of this miRNA in patients, with a 5-year OS of $45.7 \%$ and $70.9 \%$, respectively $(P=0.002$; shown in Figure 2$)$.

Univariate and multivariate analyses were utilized to evaluate whether the miR-126 expression level and various clinicopathological features were independent prognostic parameters of patient outcomes. Multivariate analysis revealed that miR-126 expression $(\mathrm{HR}=3.97$, 95\% CI: 2.01-20.22; $P=0.003$ ), distant metastasis ( $\mathrm{HR}=$ 4.78, 95\% CI: 2.31-20.12; $P=0.004)$, and FIGO stage $(\mathrm{HR}=2.12$, 95\% CI: $1.68-18.29 ; P=0.001)$ were independently associated with the overall survival (shown in Table 2).

\section{Discussion}

Accurate prediction of the prognosis for the individual patient with cervical cancer is of great importance, and molecular biomarkers that could be served as prognostic markers would be useful in determining an individualized treatment plan for a cervical cancer patient. However, the biomarkers used in this tumor group today are not satisfactory, and it is needed to exploit additional markers to fine-tune this process.

MiRNAs, a class of naturally occurring, non-coding, short single stranded RNAs, are deregulated in cancer, and they are involved in malignant transformation and tumor development. In recent years, numerous studies have shown aberrant expression of miRNAs in human cancers, including cervical cancer, some of which function as tumor suppressor genes or oncogenes. Due to their tissue-and disease-specific expression patterns and tremendous regulatory potential, miRNAs are being identified as diagnostic and prognostic biomarkers, as well as additional therapeutic tools [16,17]. As more and more studies report the relationships between miRNAs and cervical cancer, its potential as novel biomarkers in cervical cancer is growing [18-20].

MiR-126, derived from a common precursor structure located within the epidermal growth factor-like domain 7 (EGFL7) gene, is frequently down-regulated in a variety of malignancies and acts as a potential tumor suppressor [10-12]. Previous studies have reported that miR-126 may play a role in tumorigenesis and growth by regulating the vascular endothelial growth factor (VEGF)/phosphoinositol 3-kinase (PI3K)/AKT signaling pathways in human breast cancer [21]. Additionally, this miRNA may function as a tumor suppressor, with Crk as a direct target, in gastric cancer [22] and via the 


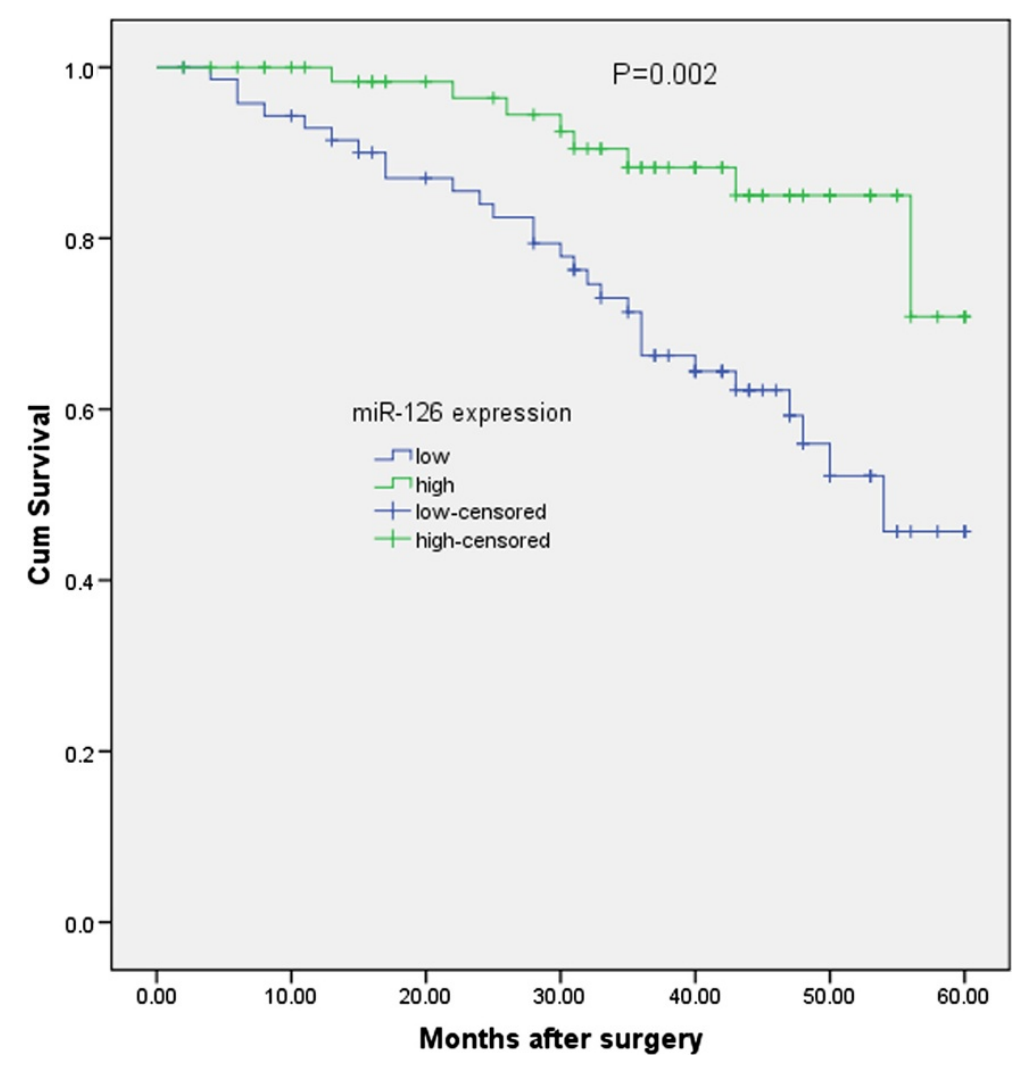

Figure 2 Survival analysis of the miR-126 expression levels with overall survival of patients with cervical cancer after surgery.

regulation of ADAM9b in pancreatic cancer [23]. miR126 may also play a role in angiogenesis in ischemia [24], and has also been reported to enhance the sensitivity of non-small cell lung cancer(NSCLC) cells to anticancer agents by targeting VEGF-A [25]. Together, these previous studies have demonstrated the important role of miR-126 in various cancers. Moreover, low expression of miR-126 has been found to be correlated with poor prognosis in patients with breast cancer, adult $\mathrm{T}$ cell leukemia, CRC and malignant mesothelioma [13-15]. Previously, miR-126 expression in cervical cancer tissues

Table 2 Multivariate Cox's hazards model analysis for prognostic factors

\begin{tabular}{llll}
\hline Variable & Hazard ratio & $\mathbf{9 5 \% ~ C l}$ & P value \\
\hline Age $(\mathrm{y})$ & 1.02 & $0.29-2.88$ & 0.66 \\
HPV & 1.28 & $0.79-2.44$ & 0.11 \\
Tumor Size $(\mathrm{cm})$ & 2.11 & $0.81-3.19$ & 0.09 \\
Tumor histology & 0.91 & $0.25-1.91$ & 0.81 \\
Lymphatic invasion & 3.14 & $0.88-19.23$ & 0.06 \\
Distant metastasis & 4.78 & $2.31-20.12$ & 0.004 \\
FIGO stage & 2.12 & $1.68-18.29$ & 0.001 \\
Histological grade & 5.11 & $0.91-17.89$ & 0.07 \\
miR-126 expression & 3.97 & $2.01-20.22$ & 0.003 \\
\hline
\end{tabular}

was found to be significantly decreased compared with that in normal cervical tissues. Yu et al. found that miR126 was able to suppress the proliferation of cervical cancer cells and alters cell sensitivity to the chemotherapeutic drug bleomycin [26]. However, the clinical significance and prognostic value of miR-126 in cervical cancer have not been investigated. In the present study, we showed that miR-126 was significantly downregulated in cervical cancer tissues for the first time. Decreased miR-126 expression in cervical cancer was found to be significantly associated with lymphatic invasion, distant metastasis, FIGO stage, and histological grade, suggesting that miR-126 might be involved in the carcinogenesis and metastasis of cervical cancer. More importantly, we proved that patients with a lower expression of miR-126 tended to have shorter survival than patients with higher levels. Furthermore, multivariate analysis revealed that miR-126 expression was independently associated with the OS, indicating that lower miR126 level was a marker of poor prognosis for patients with cervical cancer.

\section{Conclusions}

In conclusion, this study indicated that down-regulation miR-126 was associated with tumor progression and poor prognosis in cervical cancer and was identified for 
the first time as an independent poor prognostic factor for patients with cervical cancer. Further study with a larger case population is needed to confirm the prognostic value of miR126 expression in cervical cancer.

\section{Competing interests}

The authors declare that they have no competing interests.

\section{Authors' contributions}

YY and LC designed the study and drafted the manuscript; YY, KLS, HC, and LC carried out the experiments and performed the data analysis. All authors read and approved the final manuscript.

\section{Author details}

'Department of Gynecology, Qingdao Municipal Hospital, No.1 Jiaozhou Road, Qingdao 266011, People's Republic of China. ${ }^{2}$ Department of Gynecology, People's hospital of Sanya, Sanya 572000, China. ${ }^{3}$ Department of Pathology, Qingdao Municipal Hospital, Qingdao 266011, China.

Received: 29 September 2014 Accepted: 10 November 2014 Published online: 31 December 2014

\section{References}

1. Siegel R, Ma J, Zou Z, Jemal A: Cancer statistics, 2014. CA Cancer J Clin 2014, 64(1):9-29.

2. Waggoner SE: Cervical cancer. Lancet 2003, 361(9376):2217-2225.

3. Yanokura M, Banno K, Kobayashi Y, Kisu I, Ueki A, Ono A, Masuda K, Nomura H, Hirasawa A, Susumu N, Aoki D: MicroRNA and endometrial cancer: Roles of small RNAs in human tumors and clinical applications (Review). Oncol Lett 2010, 1(6):935-940.

4. Kelly BD, Miller N, Healy NA, Walsh K, Kerin MJ: A review of expression profiling of circulating microRNAs in men with prostate cancer. BJU Int 2013, 111(1):17-21.

5. Nair VS, Maeda LS, loannidis JP: Clinical outcome prediction by microRNAs in human cancer: a systematic review. J Natl Cancer Inst 2012, 104(7):528-540.

6. Jiang YW, Chen LA: microRNAs as tumor inhibitors, oncogenes, biomarkers for drug efficacy and outcome predictors in lung cancer (review). Molecular Med Reports 2012, 5(4):890-894.

7. Lages E, Ipas H, Guttin A, Nesr H, Berger F, Issartel JP: MicroRNAs: molecular features and role in cancer. Front Biosci 2012, 17:2508-2540.

8. Kwak PB, Iwasaki S, Tomari Y: The microRNA pathway and cancer. Cancer Sci 2010, 101(11):2309-2315.

9. Sotiropoulou G, Pampalakis G, Lianidou E, Mourelatos Z: Emerging roles of microRNAs as molecular switches in the integrated circuit of the cancer cell. RNA 2009, 15(8):1443-1461.

10. Walter BA, Valera VA, Pinto PA, Merino MJ: Comprehensive microRNA profiling of prostate cancer. J Cancer Educ 2013, 4(5):350-357.

11. Wang X, Tang S, Le SY, Lu R, Rader JS, Meyers C, Zheng ZM: Aberrant expression of oncogenic and tumor-suppressive microRNAs in cervical cancer is required for cancer cell growth. PLoS One 2008, 3(7):e2557.

12. Tavazoie SF, Alarcon C, Oskarsson T, Padua D, Wang Q, Bos PD, Gerald WL, Massague J: Endogenous human microRNAs that suppress breast cancer metastasis. Nature 2008, 451(7175):147-152.

13. Liu Y, Zhou Y, Feng X, Yang P, Yang J, An P, Wang H, Ye S, Yu C, He Y, Luo $\mathrm{H}$ : Low expression of microRNA-126 is associated with poor prognosis in colorectal cancer. Genes Chromosom Cancer 2014, 53(4):358-365.

14. Ishihara K, Sasaki D, Tsuruda K, Inokuchi N, Nagai K, Hasegawa H, Yanagihara K, Kamihira S: Impact of miR-155 and miR-126 as novel biomarkers on the assessment of disease progression and prognosis in adult T-cell leukemia. Cancer Epidemiol 2012, 36(6):560-565.

15. Tomasetti M, Staffolani S, Nocchi L, Neuzil J, Strafella E, Manzella N, Mariotti L, Bracci M, Valentino M, Amati M, Santarelli L: Clinical significance of circulating miR-126 quantification in malignant mesothelioma patients. Clin Biochem 2012, 45(7-8):575-581.

16. Shen J, Stass SA, Jiang F: MicroRNAs as potential biomarkers in human solid tumors. Cancer Lett 2013, 329(2):125-136.

17. Allegra A, Alonci A, Campo S, Penna G, Petrungaro A, Gerace D, Musolino C: Circulating microRNAs: new biomarkers in diagnosis, prognosis and treatment of cancer (review). Int J Oncol 2012, 41(6):1897-1912.
18. Wang $N$, Zhou Y, Zheng L, Li H: MiR-31 is an independent prognostic factor and functions as an oncomir in cervical cancer via targeting ARID1A. Gynecol Oncol 2014, 134(1):129-137.

19. Zhao S, Yao D, Chen J, Ding N: Circulating miRNA-20a and miRNA-203 for screening lymph node metastasis in early stage cervical cancer. Genetic Testing Molecular Biomarkers 2013, 17(8):631-636.

20. Shen SN, Wang LF, Jia YF, Hao YQ, Zhang L, Wang H: Upregulation of microRNA-224 is associated with aggressive progression and poor prognosis in human cervical cancer. Diagn Pathol 2013, 8:69.

21. Zhu N, Zhang D, Xie H, Zhou Z, Chen H, Hu T, Bai Y, Shen Y, Yuan W, Jing $\mathrm{Q}$, Qin Y: Endothelial-specific intron-derived miR-126 is down-regulated in human breast cancer and targets both VEGFA and PIK3R2. Mol Cell Biochem 2011, 351(1-2):157-164.

22. Feng R, Chen X, Yu Y, Su L, Yu B, Li J, Cai Q, Yan M, Liu B, Zhu Z: miR-126 functions as a tumour suppressor in human gastric cancer. Cancer Lett 2010, 298(1):50-63.

23. Hamada S, Satoh K, Fujibuchi W, Hirota M, Kanno A, Unno J, Masamune A, Kikuta K, Kume K, Shimosegawa T: MiR-126 acts as a tumor suppressor in pancreatic cancer cells via the regulation of ADAM9. Molecular cancer research: MCR 2012, 10(1):3-10.

24. van Solingen $C$, de Boer HC, Bijkerk R, Monge M, van Oeveren-Rietdijk AM, Seghers L, de Vries MR, van der Veer EP, Quax PH, Rabelink TJ, van Zonneveld AJ: MicroRNA-126 modulates endothelial SDF-1 expression and mobilization of Sca-1(+)/Lin(-) progenitor cells in ischaemia. Cardiovasc Res 2011, 92(3):449-455.

25. Zhu X, Li H, Long L, Hui L, Chen H, Wang X, Shen H, Xu W: miR-126 enhances the sensitivity of non-small cell lung cancer cells to anticancer agents by targeting vascular endothelial growth factor A. Acta Biochim Biophys Sin 2012, 44(6):519-526.

26. Yu Q, Liu SL, Wang H, Shi G, Yang P, Chen XL: miR-126 Suppresses the proliferation of cervical cancer cells and alters cell sensitivity to the chemotherapeutic drug bleomycin. Asian Pacific journal of cancer prevention : APJCP 2013, 14(11):6569-6572.

\section{Submit your next manuscript to BioMed Central and take full advantage of:}

- Convenient online submission

- Thorough peer review

- No space constraints or color figure charges

- Immediate publication on acceptance

- Inclusion in PubMed, CAS, Scopus and Google Scholar

- Research which is freely available for redistribution 